\title{
Big questions about the universe
}

\author{
Magda Stavinschi \\ Astronomical Institute of the Romanian Academy, \\ Bucharest, str. Cuţitul de Argint 5, 040557 Bucharest, Romania \\ email: magda_stavinschi@yahoo.fr
}

\begin{abstract}
Astronomy is not only a branch of science but also an important part of the culture and civilisations of peoples. Starting with archeoastronomy to the present day, it has always contributed to a better understanding of life, of humanity. After 400 years of modern astronomy, it still addresses major problems such as: Why there is something rather than nothing? Why is nature comprehensible to humans? How is cosmos related to humanity? Do multiverses exist? Is there life on other planets? Are we alone in the universe? Does the universe have a beginning? If so, what does it mean? How did the universe originate? All these questions are a challenge for interdisciplinary and transdisciplinary investigations, for philosophers, physicists, cosmologists, mathematicians, theologians. The new insights gained by pursuing in depth these common investigations will shape the society we live in and have important consequences on the future we are creating.
\end{abstract}

Keywords. Philosophy, sociology, religion

Four centuries have already elapsed since Galileo directed a telescope to the sky, since humankind has had a new vision about the universe, four centuries in which astronomy has registered a remarkable progress. The universe frontiers have advanced and so have the frontiers of our knowledge. Many puzzles have been solved, many questions have been answered, but new ones keep rising at the horizon, questions which science cannot solve, at least for the time being.

The rôle of the astronomers is precisely to identify them, to try to solve them and why not, to work together with other scientists in order to find an answer to them. Actually, astronomy is not only a science, as it has been defined. In its broadest sense, science (from the Latin scientia, meaning "knowledge") refers to any systematic knowledge-based or prescriptive practice which is capable of resulting in a prediction or predictable type of outcome. In this sense, science often coincides with art, which may contain elements of practice (praxis) that, because of uncontrollability or undefinability, have not been reduced to a reproducible recipe.

If science can be so complex in general, astronomy is even more complex. Its study implies an intellectual and spiritual movement of scientists, scholars, philosophers, artists, and citizens across the globe. Moreover it means education and outreach, from the youngest ages to the oldest ones. Everybody expects to receive the most pertinent explanations from scientists, and the astronomers to give the answers about the universe.

Starting with archeoastronomy to the present day, astronomy has always contributed to a better understanding of life, of humanity. Which are the most frequent and difficult questions of the present?

- Why there is something rather than nothing?

- Why is nature comprehensible to humans?

- How is cosmos related to humanity? 
- Does multiverses exist?

- Is there life on other planets?

- Are we alone in the universe?

- Does universe have a beginning? If so, what does it mean?

- How did the universe originate?

All these questions are a challenge for interdisciplinary and transdisciplinary investigations, for philosophers, physicists, cosmologists, mathematicians, theologians.

The new insights gained by pursuing in depth this common investigation will shape the society we live in and have important consequences on the future we are creating. Through the educational programs and publications, IAU Commission 46 Education and Development draws together many diverse fields of knowledge and their representative experts to tackle today's complex challenges. In pursuit of this vision, we are dedicating our best efforts, and we ask you -we urge you- to join us at this most pivotal moment in our history, offered by the IYA 2009.

It is imperative that we learn how to think, teach, and act in this way if we are to have the opportunity for a better future -more just, more secure, more convivial, and wiser. We would like to mention in the first place the most striking example: there are many schools in the world where religion is taught (as in Romania). The pupil gets two kinds of information concerning the birth of the universe: one from the lessons of natural science (actually, much too reduced lately) and another one from the Genesis, from the Bible. Who should explain to them which is actually the truth? Or, more correctly, which is the difference between scientific information and the symbols of the Bible or of the other holy books?

Another question which has troubled society lately is the rumors about the end of the world. The newspapers abound in "terrible discoveries about the apocalypse of 2012". What is truth in the Mayas calendar, what do we know about the end of the world and about that of our planet in the first place? These are questions which should pass from the world of science to that of the general public.

Nevertheless, how will the universe end? By the Big Crunch, by the Big Chill, or by the Big Rip? The latest information launched by the press, the scientific one included, envisages the possibility of the existence of other universes, of the multiverse. Specialists in quantum physics, like David Deutsch, a physics professor at Oxford University and winner of the prestigious Dirac prize, assert that they really exist and that this kind of phenomena which are difficult to explain can be easily explained intuitively. Metaphysicians, like David Lewis, one of the most renowned figures of contemporary analytical philosophy, also speak about the real existence of possible worlds. Naturally, many other pieces of information can be picked up from the fantastic-scientific literature.

Last but not least there are the questions concerning the relation between the heavenly bodies and our own destiny. From mentioning great personalities who were also astrologers (Kepler, we believe, is the most illustrative example) up to the use of the most sophisticated calculation techniques to prove that astrology is actually "a science", astronomers have to give the answers to those who expect the scientific truth from us.

A fundamental question was raised recently by the laureate of the John Templeton Foundation in 2008, Professor Michael Heller. Does the Universe need to have a cause? is the question he raises:

"Various processes in the universe can be displayed as a succession of states in such a way that the preceding state is a cause of the succeeding one. If we look deeper at such processes, we see that there is always a dynamical law prescribing how one state should generate another state. But dynamical laws are expressed in the form of mathematical equations, and if we ask about the cause of the universe we 
should ask about a cause of mathematical laws. By doing so we are back in the Great Blueprint of God's thinking the universe. The question on ultimate causality is translated into another of Leibniz's questions: "Why is there something rather than nothing?" (from his Principles of Nature and Grace). When asking this question, we are not asking about a cause like all other causes. We are asking about the root of all possible causes."

His is not the single voice to this effect. Recently, in Beijing took place the congress organized by John Templeton Foundation and the Chinese Academy of Sciences. New Vision 400: Engaging Big Questions in Astronomy and Cosmology Four Hundred Years after the Invention of the Telescope (October, 12-15, 2008). The public lectures were: From the Language of Heaven to the Rationale of Matter (Tsung-Dao Lee, Columbia University), The Impact of Modern Telescope Development on Astronomy (Riccardo Giacconi, Johns Hopkins University), Searching for Other Earths and Life in the Universe (Geoff Marcy, University of California, Berkeley). Another conference approaching issues at the frontiers of knowledge was Metanexus Conference 2009 - Cosmos, Nature, Culture: A Transdisciplinary Conference (July 18 - 21, 2009 Phoenix, Arizona). Philosophers, biologists, physicists, cosmologists, neuroscientists, cognitive scientists, theologians, scholars in religious studies, and other researchers and educators discussed these and other profound questions of cosmos, nature, and culture in a rapidly evolving and complex world.

Why a transdisciplinary approach? Transdisciplinarity concerns that which is at the same time between the disciplines, across the different disciplines, and beyond all discipline. The word was introduced for the first time by Jean Piaget in 1970, but it acquired an extraordinary scope due to the French scholar of Romanian origin Basarab Nicolescu, who defines transdisciplinarity via three methodological postulates: existence of Levels of Reality and Levels of Perception, included middle logic and complexity. This is the context in which astronomers and physicists from South-East Europe, gathered many times at the UNESCO-BRESCE meetings (Regional Bureau for Science and Culture in Europe) raised the question of approaching the fundamental problems of the universe in the context of the latest scientific discoveries, but also in that of the philosophical approaches. This is what we set out to approach within the project named Science and Spiritual Information: Big Questions about the Universe.

In the first place we have started from the experience already gained in Romania through previous projects in which science, philosophy, religion, art have clashed in approaching some fundamental topics which concern humankind today. The present project has a dual objective: to contribute to creating a true regional dimension concerning transdisciplinary research about the fundamental questions and a national component designed to train a large number of high school teachers as well as a small number of chosen students. The research group designed to study a certain number of fundamental issues will be composed of researchers coming from at least six different countries, while the training program within Romania will reach 1,500 high school teachers and 100 post doctoral students.

A multidisciplinary group of international researchers will be set up with people from South-East Europe. This group of researchers will study the following questions:

- physical causality and causality of causes

- absurdity or purpose and hope in the universe

- rationality of the universe

- physical information and spiritual information

- social impact of spiritual information.

This non-exhaustive list of multi-, inter- and transdisciplinary subjects will also serve as research themes for post-doctoral university summer school programmes. 
The research group composed of researchers from six different countries and representing diverse scientific disciplines as well as philosophical and theological ones, will meet ten times per year in Romania and in several of the countries of SE Europe. Specialised articles concerning each of the themes are planned as well as a collective work and a documentary which will treat some of the subjects studied (but not all) with visual illustrations and which will be broadcast by one of Romania's main television stations.

Concerning the post-doctoral essay contest, a significant advertising campaign will be conducted including the purchase of advertising space in large circulation newspapers and magazines as well as in the specialized press. Moreover, building on an experience already conducted at Craiova by one of the groups of the current project, and to the European program Hands on Universe, a training program will be set up for 1,500 teachers. It will train them in the way to approach the problems posed by bringing together science and spirituality and which concern the origins of the universe, the nature of the human being, the nature of mathematics, the evolution of life, etc. Several regional poles will be created permitting several hundred teachers to be gathered locally to benefit from these trainings.

Acquiring new spiritual information requires the simultaneous confrontation of scientific results with the intuitions of different spiritual traditions. It also requires training a new generation of researchers open to questions which are, by definition, multi-, inter- and transdisciplinary in as much as spiritual training cannot be obtained by a reductionist, technical approach confined within the limits of a single discipline. The current project responds to these two preoccupations: its international dimension aims at mobilising the intellectual and spiritual resources of different countries located in this region of Europe to study a certain number of fundamental questions by multidisciplinary teams. At the same time, it will ensure a general training of high school teachers as well as a more specialised t raining for a certain number of post-doctoral students with the capacity of forming the core of a new group of public opinion leaders concerning these questions. It is in this way that it responds to donor intent by contributing in Romania and in the neighboring countries, to an intellectual structure favourable to the development over the long term of new spiritual information.

These are only some of the means through which the astronomers, together with other scientists, are trying to find answers to ever deeper problems facing humankind today. 